
\title{
25 Research Square \\ Lymphoepithelioma-like cholangiocarcinoma in middle-aged woman with Epstein-Barr Virus infection: a case report and review of the literature
}

\section{Xuequan Cao}

taizhou central hospital(taizhou university hospital)

Hongsheng Lu

taizhou central hospital(taizhou university hospital)

Zhengying Ruan

taizhou central hospital(taizhou university hospital)

Luqing Zhou ( $\nabla$ zhoulq9257@tzzxyy.com )

Xiaobo Cai

taizhou central hospital(taizhou university hospital)

\section{Case Report}

Keywords: Lymphoepithelioma-like cholangiocarcinoma, Intrahepatic cholangiocarcinoma, Lymphoepithelioma-like carcinoma, EBER

Posted Date: March 11th, 2020

DOl: https://doi.org/10.21203/rs.3.rs-16786/v1

License: (c) (i) This work is licensed under a Creative Commons Attribution 4.0 International License. Read Full License 


\section{Abstract}

\section{Background}

Lymphoepithelioma-like carcinoma(LELCC) is a rare variant of intrahepatic cholangiocarcinoma (ICC), LELCC is composed of prominently reactive lymphoid infiltration and scattered malignant epithelial cells, which may lead to misdiagnosis of pathologist, especially on rapid freezing during operation. Recognizing the tumor is important to avoid misdiagnosis.

Case presentation

We report a case of LELCC in a 53-year-old Chinese female patient with EBV infection, who was found to have a nodules in the junction of the left and right lobes of the liver on abdominal Computed tomography(CT) and magnetic resonance imaging(MRI). Microscopically, the boundary of the tumor was clear with incomplete fibrous capsule around the tumor, the tumor was arranged in a nest or tubular gland, tumor cells were cubic, with vesicular nuclei, prominent nucleoli, rare mitotic figures, and abundant lymphoid infiltration and lymphofollicular formation. Immunohistochemically, tumor cells were diffusely positive for biliary-type cytokeratins (CK7, CK19), CK8,CK18,Ki-67 proliferation index (about 20\%) and negative for CK20, polyclonal carcinoembryonic antigen(CEA) and CD34, hepatic marker (HepPar-1), The tumor-infifiltrating lymphocytes are predominant for CD3 and CD45RO-positive T cells, CD20 and CD79a are positive scattered in the germinal center and surrounding areas. In situ hybridization, EBV-encoded RNA (EBER) was strongly positive in tumor cells.

\section{Conclusions}

The diagnosis of LELCC should be combined with histopathology, immunohistochemistry and EBER detection. There are limited literature reports on the pathological diagnosis and differential diagnosis, additional studies will be needed to needed to clarify the clinicopathologic features, immunophenotype, and differential diagnosis of this disease. It is necessary to master the diagnostic criteria to avoid misdiagnosis for pathologist.

\section{Background}

Lymphoepithelioma-like carcinoma (LELC) is a malignant tumor resembling undifferentiated nasopharyngeal carcinoma (NPC), which is histologically composed of undifferentiated epithelial cells with prominent lymphoid infiltration[1]. LELC have been reported in varios organs including salivary gland, skin, lung,the stomach, and liver and so on[2-7]. Liver is a rare site of occurrence. In recent years, more and more cases have been reported with the further understanding of LELC[8]. LELC in the liver can be divided into lymphoepithelioma-like hepatocellular carcinoma (LELHCC), lymphoepithelioma-like cholangiocarcinoma (LELCC) and mixed LELHCC and LELCC [8-9]. LELCC, which is defined as tumors arisen in the hepatic tract and composed of undifferentiated epithelial cells arranged in gland forming, sheets, or cords with a dense lymphoid infiltration, is a rare variant of intrahepatic cholangiocarcinoma 
(ICC) $[1,10]$. Ling et al [10] retrospectively analyzed 40 cases of LELCC and found $80.0 \%$ of these cases were related to EBV. To date, only 29 cases of LELCC with Epstein-Barr Virus(EBV) Infection have been reported in the English literature [11], in addition, the diagnosis and differential diagnosis of LELCC are rarely reported $[1,11]$. The diagnosis and differential diagnosis of this extremely rare tumor are important for its identification. This paper reports a case of LELCC with EBV infection with the review of the literature, and explores its clinicopathological characteristics, immunophenotype, molecular genetic features and differential diagnosis, in order to improve the understanding of pathologists on this tumor.

\section{Case Presentation}

A 53-year-old female patient was admitted to the hospital for discovering liver mass lesions for 17 days in February 2019. She had no abdominal pain, bloating, and no chills, fever, and other discomforts. She had recently lost weight. Physical examination: consciousness, no yellow staining in the skin and sclera, no palm in the liver and spider nevus; no auscultation of cardiopulmonary auscultation. Abdomen was flat, no varicose veins of the abdominal wall, no tenderness and rebound pain, no more than liver and spleen ribs. Pre-operative laboratory tests revealed elevated Fasting glucose $(6.87 \mathrm{mmol} / \mathrm{L}$; range $3.89 \llbracket 6.1 \mathrm{mmol} / \mathrm{L})$, triglyceride $(2.42 \mathrm{mmol} / \mathrm{L} ;<1.7 \mathrm{mmol} / \mathrm{L})$, all other indicators are normal. Tumor markers including CEA, CA125, CA199 are normal, The patients was positive for hepatitis B virus surface antibody $(\mathrm{HbsAb})$, and negative for hepatitis $B$ virus surface antigen( $\mathrm{HBsAg})$, hepatitis $B$ virus Core antibodies(HbcAb), Hepatitis B virus e antigen (HBeAg) and Hepatitis B virus e antibody(HBeAb); Hepatitis $C$ virus antibodies(HCV-Ab), human immunodeficiency virus antibodies(HIV-Ab), and Treponema pallidum antibodies(TP-Ab) are negative.

Direct enhanced CT in the upper abdomen revealed round, lower-density mass in the junction of the left and right lobes of the liver, with measuring $1.9 \mathrm{~cm}$ in diameter (Fig. 1a) and a CT value of $51.8 \mathrm{HU}$.The margins are vague, and lesions have a low density in the arterial stage after enhancement, the CT value is about $74 \mathrm{HU}$, and lesions showed low density in the portal stage and delayed stage. CT diagnosis was the nodule in the junction of the left and right lobes of the liver, and small liver cancer could not be ruled out, MRI plain scan + enhanced examination was recommended. No abnormalities in gallbladder, pancreas, spleen size and density are observed. Abdominal MRI plain scan + enhanced showed abnormal nodular signals ( $\mathrm{T} 1$ low signal intensity, T2 high signal intensity, DW1 high signal intensity)with an estimated magnitude of $1.7 \mathrm{~cm} \times 1.4 \mathrm{~cm}$ in the junction of the left and right liver lobes. After enhancement, The lesion showed enhancement in arterial phase and faded signal in portal and delayed phase(Fig. 1b). MRI diagnosis was the nodule in the junction of the left and right lobes of the liver, which is very likely considered to be hepatocellular carcinoma (HCC). The size and signal of gallbladder and spleen were normal in the scanning range, and no enlarged lymph nodes were found in the retroperitoneum. The patient underwent laparoscopic liver tumor resection + cholecystectomy in our hospital. During the operation, an exophytic mass, which about $1.8 \mathrm{~cm} \times 1.5 \mathrm{~cm}$ in size and soft in texture, was found on the surface of the liver near the bottom of the gallbladder, Postoperative specimens were examined for pathology. 
Grossly, part of the liver lobes were resected, with the size of $6 \mathrm{~cm} \times 5 \mathrm{~cm} \times 3 \mathrm{~cm}$. A mass with the size of $2 \mathrm{~cm} \times 1.5 \mathrm{~cm} \times 1.3 \mathrm{~cm}$ was seen under the capsule,. The section of the mass was grayish-white, soft and had a relatively well demarcated margin(Fig. 1c),and the section of the remaining liver tissue was grayishyellow and slightly firm.

Microscopically, the boundary of the tumor was clear with incomplete fibrous capsule around the tumor, the tumor was arranged in a nest or tubular gland with abundant lymphoid infiltration with lymphofollicular formation(Fig. 1d). tumor cells were cubic, large in size, rich in cytoplasm and eosinophilic,with vesicular nuclei, prominent nucleoli, and a syncytial cytoplasmic appearance, rare mitotic figures(Fig. 1e).

Immunohistochemically, tumor cells were diffusely positive for biliary-type cytokeratins (CK7, CK19) (Fig. 1f,g), CK8,CK18, Ki-67 proliferation index (about 20\%) and negative for CK20, polyclonal carcinoembryonic antigen(CEA) and CD34, CD56, hepatic marker (HepPar-1), The tumor-infifiltrating lymphocytes are predominant for CD3(Fig. 1h) and CD45RO-positive T cells, CD20(Fig. 1i) and CD79a are positive scattered in the germinal center and surrounding areas.

In situ hybridization for EBV-encoded RNA (EBER) showed strongly positive nuclear signal in tumor cells(Fig. 1j),but not in the surrounding non-neoplastic liver tissue.

Based on these histopathological, immunohistochemical and in situ hybridization findings, a diagnosis of EBV-associated LELCC was made.

The patients were followed up for 12 months without any radiotherapy or chemotherapy. The liver, gallbladder, pancreas, and bilateral renal enhanced CT were reviewed every three months within one year after the operation. The fourth review was liver enhanced MRI. No recurrence or metastasis was found. At present, the follow-up is continuing.

\section{Discussion And Conclusions}

Epidemiology and clinical characteristics: EBV-associated ICC is very rare and can be divided into 3 types: LELC, conventional-type ICC and LELCC on the basis of the tumor cellular differentiation, and host cellular immune responses in the tumors[12].Indicating that LELCC, which was first reported by Jeng et al[13] in 2001 , is a rare variant of ICC. Literature reported, EBER in situ hybridisation was positive in $70 \%$ of LELCC cases, while conventional cholangiocarcinomas are not associated with EBV infection[13] $\mathbb{A}$ According to previous studies, 26 cases of LELCC was analyzed, found that $73.1 \%$ (19 of 26) patients were EBVpositive $[1,8]$.These results suggested that LELCC is highly associated with Epstein-Barr virus (EBV) infection [14]囚and EBV infection might promote the development of LELCC. Hepatitis B virus (HBV) and hepatitis $\mathrm{C}$ virus (HCV) has been confirmed as one of the etiologic factors for ICC[15]. However,only $26.9 \%$ (7 of 26) and $7.7 \%$ (2 of 26) LELCC patients were HBV and HCV positive, respectively. This low incidence of HBV and HCV infection suggested that these two viruses might not be significant factors in the pathogenesis of LELCC[8].Despite these findings, the molecular mechanism of EBV, HBV and HCV remain 
unknown in the tumorigenesis and development of LELCC. The published literatures reported that majority of LELCC patients were discovered in the Asian countries[11, 13, 16]. LELCC with EBV infection tends to occur in middle-aged women with a female:male ratio of more than 3:1, the range of age was 19 to 71 years (mean age, 53.39 years) [8-12]. No preference in the tumor location was found between the left and the right hepatic lobe[17]. The size of LELCC tumors ranged from 1.2 to $7.3 \mathrm{~cm}$ in diameter (mean, $3.3 \mathrm{~cm}$ ) $[12,18]$.Clinical symptoms were nonspecifific, usually found incidentally or abdominal fullness and abdominal pain[10]. In our study,EBV were positive in situ hybridization and HBV and HCV were both negative in a Chinese middle-aged woman, Clinical information was similar to previous reports. Because clinical symptoms and laboratory tests are nonspecifific for diagnosing LELCC, Imaging examination including CT, MRI, and the B-mode ultrasound and contrast-enhanced ultrasound (CEUS) is helpful for for early diagnosing of LELCC[10, 18-19].

Pathological features: Grossly,majority of these tumors were well demarcatedand with the sizes ranged from 1.2 to $7.3 \mathrm{~cm}$ in diameter, The sectioned surface showed grayish-white or white-tan or yellowish-

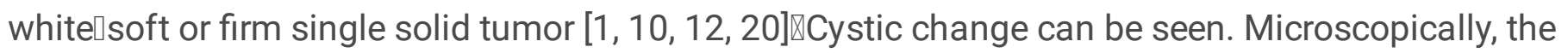
boundary of the tumor is clear, and there is an incomplete fibrous capsule around the tumor, Small tumor cells were seen outside the capsule.The tumors with varied glandular differentiation were adenocarcinomas, which were characterized by tubular, trabecular, or cord-like patterns with variable degrees of differentiation】tumor cells were cubic, large in size, rich in cytoplasm and eosinophilic $\square T h e$ nuclei was round or oval with an irregular nuclear membrane $\llbracket$ vesicular nuclei, prominent nucleoli, and a syncytial cytoplasmic appearance, rare mitotic figures. There are extensive lymphoplasmacytic infiltration in the stroma, Epithelioid granulomas and numerous lymphoid follicles could be seen. Occasionally, obvious granulomatous reactions and many multinucleated giant cells coexisted[11] .

Immunophenotype: Immunohistochemical staining showed that tumor cells were diffusely positive for AE1/AE3, epithelial membrane antigen (EMA), biliary-type cytokeratins (CK7, CK19) CK8,CK18, and biliary lineage markers (EpCAM and CA19-9) and negative for CK20, AFP, polyclonal carcinoembryonic antigen(CEA), and hepatic marker (HepPar-1 and glypican 3). The lymphoid infiltrate was positive for $\mathrm{CD} 3$ ( $T$ cell marker) and $\mathrm{CD} 20$ (B cell marker). According to the literature, More than half of tumors show a mutation-associated p53 immunohistochemistry pattern. The tumor-infifiltrating lymphocytes are predominantly CD3-positive $T$ cells, with focal CD20-positive B cells and CD138-positive plasma cells [9, 21, 22-23]. In our study, there are more T cells infiltrated marked by immunohistochemistry than B cells, which was similar to previous literature. However, Lee et al[24] reported that the B and T cells infiltrated in nearly equal proportions.

Molecular genetics: At present, there are few reports on molecular genetics of LELCC. Literature reported that gene hypermethylation was more frequent in LELCC than IHCC. No LELCC harboured any EGFR or KRAS mutation[25].Molecular analysis of the original periportal mass showed single-nucleotide variations in the tumour suppressor gene TP53 as well as single-nucleotide variations of uncertain significance in $\mathrm{MLH} 1$, a gene involved in DNA mismatch repair. The tumour was microsatellite stable by PCR[11]. Chiang et al [14]reported that 4 LELCCs with 0 to 5 mutations per sample. Identified mutations 
included BARD1 (allele frequency: 8.1\%), EPHA5 (5.8\%), MUC16 (7.6\%), TNFAIP3 (5.8\%), CD19 (5.6\%), PTEN (8.8\%), TET1 (7.2\%), RECQL4 (6.4\%), CD79B (10.1\%), and KDM5A (10.2\%), with all $20 \%$ of incidence. Each mutation is independent between four cases. In terms of molecular genetics testing, the genetic markers, which have diagnostic significance, have been found, and further information needs to be accumulated to clarify the cases.

Differential diagnosis: The diagnosis of LELCC should be combined with histopathology, immunohistochemistry and EBER detection, There are large cells with varied glandular differentiation and a lymphocyte-rich tumour stroma in LELCC. Therefore, liver LELCC should be distinguished from the following tumors: 1, Metastatic LELC or NPC: The patient underwent a comprehensive physical examination, laboratory examination, and auxiliary examination, and found no obvious lesions in organs other than the liver, including the nasopharynx.on the other hand, The tumour cells in NPC express cytokeratin and squamous antigens (p63 and CK5/6), The tumour cells in LELC may or may not express squamous antigens. Biliary-type cytokeratins (CK7 and CK19) was positive in this tumor, which is commonly used to diagnose LELCC and rule out NPC or LELC without glandular tubular structure in from other organs[25]. 2,LELHCC: The tumor cells are polygonal, rich in cytoplasm, eosinophilic, forming flaky or nested structures, and rarely forming adenoid differentiation. However, LELCC is often accompanied by adenoid differentiation. and HepPar-1, AFP, CEA, CK8, CK18 and other antibodies are often expressed in LELHCC\EBV were commonly positive in situ hybridization[1]. 3, Conventional-type hepatocellular carcinoma(HCC) and conventional-type intrahepatic cholangiocarcinomas (ICC) with abundant lymphoid infiltration : conventional-type HCC is pseudo-adenoid, trabecular, or nest-like arrangement, lymphocyte infiltration is mostly located around the tumor tissue, and tumor cells mostly express HepPar- 1 and CEA. Conventional-type ICC mostly has an irregular glandular tubular structure, with obvious proliferation of fibrous tissue, scattered lymphoid infiltration in the stroma, Immunohistochemically, tumor cells mostly express CK7 and CK19, In situ hybridization, ERER is mostly negative[13]. 4,Lymphoma: Lymphocytes in lymphomas are abnormal lymphocytes, immunohistochemical markers are monoclonal hyperplasia (expressing B or T cell marker), and epithelial cells are CK-negative. Special attention should be paid to identifying Hodgkin's lymphoma and anaplastic large cell lymphoma. Reed-Sternberg cells are usually seen under the microscope of Hodgkin's lymphoma, and CD15 and CD30 are positive for Reed-Sternberg cells. There are often many reactive cells around anaplastic large cell lymphoma, and some tumor cells are epithelial-like. Usually, weird nuclei, kidney-shaped or horseshoe-shaped nuclei are seen in the cells, and EMA, CD30 is positive for tumor cells. 5, Bile duct adenoma: it is composed of small bile ducts with benign appearance, which is mature and proliferative. There is no bile in the lumen, no atypia and mitosis, and there is chronic inflammatory cell infiltration. There were no dilated bile ducts in the tumor, In advanced stage, there were more collagen, fewer small bile ducts and inflammatory cells. CD56 was positive for bile duct adenoma. However, the epithelial cells of LELCC are atypical and CD56 was negative for epithelial cells. 6,0ther less likely neoplasms were also ruled out using immunohistochemistry, including inflammatory myofibroblastic tumour (Anaplastic lymphoma kinase (ALK)), malignant melanoma (HMB45 and S100) and histiocytic sarcoma (CD163). These tumours can have large cells with a lymphocyte-rich tumour stroma, but EBV were negative in situ hybridization[11] . 
Treatment and prognosis: LELCC might be associated with EBV infection, but whether EBV infection is associated with a better outcome or poorer prognosis, which is controversial. Compared to patients with EBV-negative ICCs, after surgery, patients with EBV-associated LELCCs usually have favorable outcomes[25]. However,Wang et al [18]reported that EBV infection leads to a poorer prognosis in LELCC. The role of EB infection in LELCC prognosis is still unclear, and more clinical studies are needed for further analysis. Zhang et al [26]found that Only 8 cases had recurrence in 52 cases of LELCC reported by searching English literature. Gearty et al [11] retrospectively analyzed the 29 LELCC cases with EBV infection, found that 8 patients died of the disease. Most cases were early stage tumours treated with surgical resection, including one successfully resected lymph node metastasis. These findings indicate that LELCC patients have a better prognosis. The prognosis of LELCC patients seems to be better than that of conventional-type cholangiocarcinoma at 5-year survival rate(100\% and $13.2 \%)[25]$.

In all cases reported, surgical resection was the the most effective choice of treatment, but the necessity of lymph node dissection had not been reported. However, postoperative radiotherapy, postoperative chemotherapy, or targeted therapy was rarely adopted. Some reports had suggested that postoperative radiation therapy and chemotherapy may be benefificial[24]. However, Zhang et al [26]reported that that surgery could be an effective way of treating lymph node metastasis of LELCC. In recent years, immunotherapy has become a new treatment option for many tumors. Of the one LELCC patient who had anti-PD-1 treatment after gemcitabine/ cisplatin therapy failure got a durable partial response to the antiPD-1 immunotherapy[14].Wang et al [18] found that PD-L1 levels were higher in LELCC than in ICC. Therefore, Immunotherapy of targeted PD-L1/PD-1 may be effective therapeutic strategy in EBVassociated LELCC. Because of the limited number of reported cases, no consensus on standardized treatment strategy for LELCC had been reported, treatment methods and prognosis need further observation and exploration. In our study,the patient, which did not receive any radiation and chemotherapy, was followed up for 12 months without recurrence or metastasis after hepatectomy. We will Currently continue to follow up.

In summary,EBV-associated LELCC $\llbracket$ which have a better prognosis than conventional-type CC, is a rare variant of ICC, The diagnosis depends on histopathology, immunohistochemistry and EBER detection, and the recognition and correct diagnosis of LELCC has important clinical significance for the treatment and prognosis of patients.

\section{Abbreviations}

LELCC :Lymphoepithelioma-like carcinoma; ICC: intrahepatic cholangiocarcinoma ; CT: Computed tomography; MRI: magnetic resonance imaging; CK:cytokeratins; EBER: EBV-encoded RNA; LELC :Lymphoepithelioma-like carcinoma; NPC:undifferentiated nasopharyngeal carcinoma; LELHCC: lymphoepithelioma-like hepatocellular carcinoma; $\mathrm{HbsAb}$ :hepatitis B virus surface antibody; HBsAg: hepatitis B virus surface antigen; $\mathrm{HbcAb}$ :hepatitis B virus Core antibodies; HBeAg :Hepatitis B virus e antigen; HBeAb:Hepatitis B virus e antibody; HCV-Ab:Hepatitis C virus antibodies; HIV-Ab: human 
immunodeficiency virus antibodies; TP-Ab: Treponema pallidum antibodies; HCC: hepatocellular carcinoma

\section{Declarations}

\section{Acknowledgements}

Not applicable.

\section{Funding}

The authors declare that this paper was not financially supported.

\section{Availability of data and materials}

All data for this study are presented in the manuscript.

\section{Authors' contributions}

All co-authors approved the manuscript.

\section{Authors' information}

Not applicable.

\section{Competing interests}

The authors declare that they have no competing interests.

\section{Consent for publication}

Written informed consent for publication was obtained from the patient's family.

\section{Ethics approval and consent to participate}

Not applicable

\section{References}

1. Labgaa I, Stueck A, Ward SC. Lymphoepithelioma-Like Carcinoma in Liver. Am J Pathol.2017;187(7):1438-1444.

2. Tsai $\mathrm{CC}$, Chen $\mathrm{CL}$, Hsu HC. Expression of Epstein-Barr virus in carcinomas of major salivary glands: a strong association with lymphoepithelioma-like carcinoma. Hum Pathol.1996;27(3):258-262. .

3. Ferlicot S, Plantier F, Rethers L, Bui A D, Wechsler J . Lymphoepithelioma-like carcinoma of the skin: a report of 3 Epstein-Barr virus (EBV)-negative additional cases. Immunohistochemical study of the 
stroma reaction. J Cutan Pathol .2000;27(6):306-311.

4. Castro CY, Ostrowski ML, Barrios R, Green L K, Popper H H, Powell S, et al. Relationship between Epstein-Barr virus and lymphoepithelioma-like carcinoma of the lung: a clinicopathologic study of 6 cases and review of the literature. Hum Pathol. 2001;32(8):863-872.

5. Shinozaki-Ushiku A, Kunita A, Fukayama M. Update on Epstein-Barr virus and gastric cancer (review). Int J Oncol. 2015;46(4):1421-1434.

6. Solinas A, Calvisi DF: Lessons from rare tumors: hepatic lymphoepithelioma-like carcinomas. World J Gastroenterol 2015, 21:3472e3479

7. Lin A, Alpert L, Hart J, Chapman C, Pillai A A . Lymphoepithelioma-like carcinomas - a rare variant of cholangiocarcinoma [published online ahead of print, 2020 Jan 6]. Hepatology. 2020;10.

8. Ding Y, Sun Z, You W, Zhang S , Chang C, Yan S, et al. Lymphoepithelioma-like intrahepatic cholangiocarcinoma with Epstein-Barr virus infection: report of a rare case. Ann Transl Med. 2019;7(18):497.

9. Henderson-Jackson E, Nasir NA, Hakam A, Nasir A, Coppola D . Primary mixed lymphoepitheliomalike carcinoma and intra-hepatic cholangiocarcinoma: a case report and review of literature[J]. Int $\mathrm{J}$ Clin Exp Pathol. 2010;3(7):736-741. .

10. Ling W, Lu C, Huang H, Qiu T, Lu Q , Huang C,et al. Ultrasonographic findings of intrahepatic lymphoepithelioma-like cholangiocarcinoma associated with Epstein-Barr virus: Two cases report.Medicine (Baltimore). 2019;98(3):e14206.

11. Gearty SV, Al Jurdi A, Pittman ME, Gupta R. An EBV+ lymphoepithelioma-like cholangiocarcinoma in a young woman with chronic hepatitis B. BMJ Case Rep. 2019;12(7):e229520.

12. Sun K, Xu S, Wei J, Wei J , Wang B, Owusu-Ansah K G, Wang W, et al. Clinicopathological features of 11 Epstein-Barr virus-associated intrahepatic cholangiocarcinoma at a single center in China. Medicine (Baltimore). 2016;95(40):e5069.

13. Jeng YM, Chen CL, Hsu HC. Lymphoepithelioma-like cholangiocarcinoma: an Epstein-Barr virusassociated tumor. Am J Surg Pathol. 2001;25(4):516-520.

14. Chiang NJ, Chen MH, Tsai MH, Tsai HW , Chen LT, Shan YS. Explore the mutational landscape and immune profile of EBV-associated lymphoepithelioma-like cholangiocarcinoma. HepatoBiliary Surg Nutr. 2019;8(Suppl 1):AB035.

15. Palmer WC, Patel T. Are common factors involved in the pathogenesis of primary liver cancers? A meta-analysis of risk factors for intrahepatic cholangiocarcinoma. J Hepatol.2012;57(1):69-76.

16. Huang Y, Tsung JS, Lin CW, Cheng TY. Intrahepatic cholangiocarcinoma with lymphoepithelioma-like carcinoma component. Ann Clin Lab Sci. 2004;34(4):476-80.

17. Liao TC, Liu CA, Chiu NC, Yeh YC, Chiou YY. Lymphoepithelioma-like cholangio-carcinoma: a mimic of hepatocellular carcinoma on imaging features. World J Gastroenterol. 2015;21(13):4089-4095.

18. Wang L, Dong H, Ni S, Huang D , Tan C , Chang B , et al. Programmed death-ligand 1 is upregulated in intrahepatic lymphoepithelioma-like cholangiocarcinoma. Oncotarget. 2016;7(43):69749-69759. 
19. Kim YC, Park MS, Chung YE, Kim MJ, Park Y N , Kang JH, et al. MRI findings of uncommon nonhepatocyte origin primary liver tumours with pathological correlation. $\mathrm{Br} \mathrm{J}$ Radiol. 2010;83(996):1080-1086.

20. Aosasa S, Maejima T, Kimura A, Nishiyama K, Edo H, Shinmoto H, et al. Intrahepatic Cholangiocarcinoma With Lymphoepithelioma-like Carcinoma Components Not Associated With Epstein-Barr Virus: Report of a Case. Int Surg. 2015;100(4):689-695.

21. Chen TC, Ng KF, Kuo T. Intrahepatic cholangiocarcinoma with lymphoepithelioma-like component. Mod Pathol. 2001;14(5):527-532.

22. Szekely E. Lymphoepithelioma-like cholangiocarcinoma (LELC) not associated with Epstein-Barr virus. Am J Surg Pathol. 2001;25(11):1464-1466.

23. Ishida M, Mori T, Shiomi H, Naka S, Tsujikawa T, Andoh A, et al. Non-Epstein-Barr virus associated lymphoepithelioma-like carcinoma of the inferior common bile duct.World $\mathrm{J}$ Gastrointest Oncol. 2011;3(7):111-115.

24. Lee W. Intrahepatic lymphoepithelioma-like cholangiocarcinoma not associated with epstein-barr virus: a case report. Case Rep Oncol. 2011;4(1):68-73.

25. Chan AW, Tong JH, Sung MY, Lai PB, To KF. Epstein-Barr virus-associated lymphoepithelioma-like cholangiocarcinoma: a rare variant of intrahepatic cholangiocarcinoma with favourable outcome.Histopathology. 2014;65(5):674-683.

26. Zhang JW, Yang HY, Xu YY, Sang XT, Yu SN, Huang HC, et al. Surgical treatment for metastasis from lymphoepithelioma-like cholangiocarcinoma in the liver: A case report. Medicine (Baltimore). 2018;97(19):e0666.

\section{Figures}



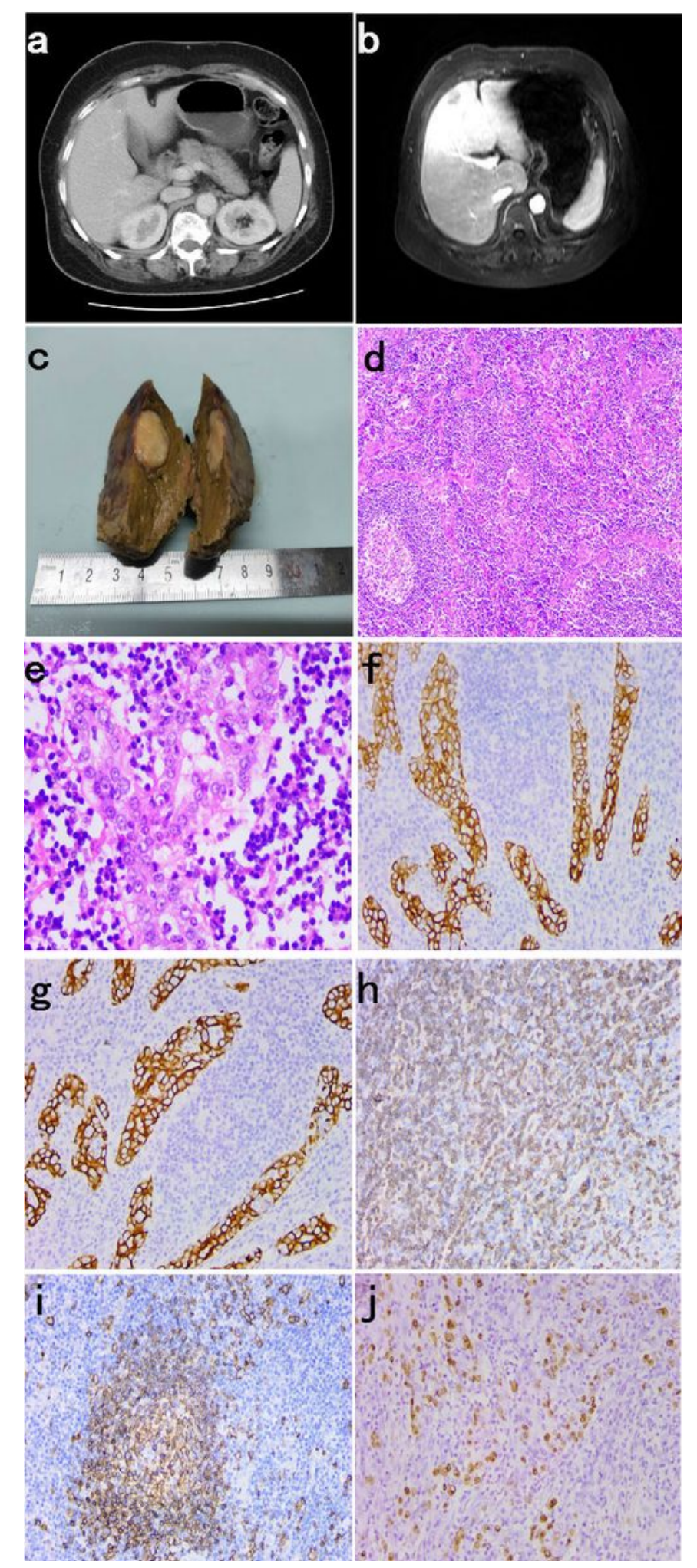

\section{Figure 1}

a A low-density nodule located in the junction of the left and right lobes of the liver, with measuring1.9 $\mathrm{cm}$ in diameter and a vague margin was also observed in the CT scan. b MRI showed abnormal nodular signals with an estimated magnitude of $1.7 \mathrm{~cm} \times 1.4 \mathrm{~cm}$ in the junction of the left and right liver lobes.c Grossly, cut surface of resected mass was greyish-white, soft, and had a relatively well demarcated margin.d Microscopically, the tumor was composed of undifferentiated epithelial cells with prominent 
lymphoid infiltration and scattered lymphoid follicles (hematoxylin and eosin stain, original magnification×100). e Tumor cells were cubic, with vesicular nuclei, prominent nucleoli, and a syncytial cytoplasmic appearance(hematoxylin and eosin stain, original magnification $\times 200$ ). $f$ CK7 was positive in the tumor cells( Immunohistochemical staining, original magnification×200). $\mathrm{g}$ CK19 was positive in the tumor cells( Immunohistochemical staining, original magnification $\times 200)$. h The tumor-infifiltrating lymphocytes were predominant for CD3-positive T cells ( Immunohistochemical staining, original magnification $\times 200$ ). i CD20 was positive for $B$ cells scattered in the germinal center and surrounding areas. (Immunohistochemical staining, original magnification $\times 200$ ). j EBV - encoded RNA (EBER) showed strongly positive in the tumor cells (EBER in situ hybridization, original magnification $\times 200$ )

\section{Supplementary Files}

This is a list of supplementary files associated with this preprint. Click to download.

- CAREchecklistEnglish2013.pdf.docx 